\title{
Metabolismo mineral em bubalinos com ingestões de diferentes níveis de fósforo
}

\author{
Nedilse Helena de Souza ${ }^{1}$, Raul Franzolin², Weber Vilas Bôas Soares ${ }^{3}$
}

\author{
1 Doutora em Zootecnia pela FZEA/USP. \\ 2 Faculdade de Zootecnia e Engenharia de Alimentos, Universidade de São Paulo, Campus de Pirassununga. \\ ${ }^{3}$ APTA, Polo Regional de Mococa, SP.
}

RESUMO - Objetivou-se avaliar os efeitos da ingestão diária de quatro níveis de fósforo (8, 12, 15 e 18 g) sobre o metabolismo de macrominerais ( $\mathrm{P}, \mathrm{Ca}, \mathrm{Mg}, \mathrm{Na}, \mathrm{K}$ e S), incluindo a ingestão, a concentração no rúmen, a taxa de passagem do líquido ruminal, a excreção nas fezes e a disponibilidade aparente. Utilizaram-se quatro bubalinos adultos com fístulas ruminais em delineamento quadrado latino $(4 \times 4)$ com dieta total constituída de cana-de-açúcar como volumoso $(85 \%)$ e concentrado formulado com um dos níveis de fósforo. Os níveis de fósforo não ocasionaram diferença significativa na concentração mineral no rúmen de nenhum mineral estudado. A concentração média de fósforo no conteúdo ruminal foi de $0,98 \%$ na matéria seca, enquanto o teor de fósforo nas rações variou de 0,12 a $0,34 \%$, comprovando alta reciclagem de fósforo pela saliva. Níveis crescentes de fósforo na dieta, variando de 8 a 18 g/animal/dia, não influenciam as disponibilidades de cálcio e magnésio. Com o nível de fósforo de $15 \mathrm{~g} /$ dia, houve melhor utilização do fósforo da dieta. A ingestão de níveis crescentes de fósforo em g/ $\mathrm{kg}^{0,75}(\mathrm{X})$ promoveu aumento linear na excreção fecal desse mineral em g/ $\mathrm{kg}^{0,75}$ (Y) e baixos valores de disponibilidade do fósforo, que pode ser estimado pela equação $\mathrm{Y}=0,03+0,610 \mathrm{X}$, o que indica deficiência desse elemento mineral na dieta para o metabolismo animal.

Palavras-chave: búfalo, minerais, rúmen

\section{Mineral metabolism in buffaloes fed different phosphorus levels}

\begin{abstract}
The objective was to evaluate the effects of daily intake of four levels of phosphorus (8, 12, 15 and $18 \mathrm{~g}$ ) on the metabolism of macro minerals ( $\mathrm{Ca}, \mathrm{P}, \mathrm{Mg}, \mathrm{Na}, \mathrm{K}$ and S), including the intake, concentration in rumen, ruminal fluid passage rate, excretion in feces and apparent availability. Four rumen-fistulated buffaloes were allotted to a $4 \times 4$ Latin Square, with total diet composed by sugar cane as forage (85\%) and concentrate formulated for each different phosphorus levels. Phosphorus levels did not caused difference in mineral concentration in the rumen of any mineral studied. Mean phosphorus concentration in the rumen content was $0.98 \%$ in the dry matter basis, whereas phosphorus content in diet ranged from 0.12 to $0.34 \%$, demonstrating high recycling of phosphorus by saliva. Increasing phosphorus levels in the diet ranging from 8 to $18 \mathrm{~g} / \mathrm{anim} /$ day did not affect the calcium and magnesium availability. Better use of phosphorus of the diet was observed at the level $15 \mathrm{~g}$ /day of phosphorus. The intake of increasing phosphorus levels in $\mathrm{g} / \mathrm{kg}^{0.75}$ (X) promoted a linear increase in fecal excretion of this mineral in $\mathrm{g} / \mathrm{kg}^{0.75}(\mathrm{Y})$, and low phosphorus availability values, which could be estimated using the regression equation $\mathrm{Y}=0.03+0.610 \mathrm{X}$, that indicate deficiency of this mineral in the diet for animal metabolism.
\end{abstract}

Key Words: buffalo, minerals, rumen

\section{Introdução}

O conhecimento do metabolismo mineral em ruminantes é de grande relevância, tendo em vista os problemas causados pela deficiência mineral, principalmente em animais criados a pasto. Segundo Torkania et al. (2000), deficiências minerais acentuadas podem ser responsáveis pela pobreza geral em determinadas regiões, onde a população depende principalmente da criação de animais ruminantes. Deficiências leves ou moderadas também podem causar prejuízos econômicos sérios, pois reduzem a produtividade dos animais e constituem obstáculo à melhoria dos rebanhos.

O metabolismo mineral na espécie bubalina não é tão conhecido como nos bovinos; há carência de informações sobre o comportamento digestivo e o aproveitamento de minerais nessa espécie animal. Estudos que forneçam essas informações são importantes para o manejo nutricional adequado, principalmente quanto ao fornecimento de dietas ideais ou alternativas para maior produtividade desta espécie. 
Conforme relatado por Barcellos (1998), a fração do fósforo da dieta que é absorvida constitui a absorção verdadeira, enquanto a diferença entre o fósforo consumido e o fósforo nas fezes representa a absorção aparente. Nos herbívoros, a rota primária de excreção de fósforo é a via fecal e consiste em importante mecanismo de controle da homeostasia do mineral. Nas fezes, o fósforo aparece em duas frações: fósforo da dieta que não foi absorvido e fósforo endógeno, o qual é principalmente de origem salivar. As perdas endógenas fecais são a forma mais importante de perda de fósforo nos ruminantes. Silva Filho et al. (2000) observaram excreção média diária de fósforo nas fezes de novilhos Nelores de 4,50; 6,35 e 8,05 g mantidos com dietas contendo fosfato bicálcico em níveis correspondentes a 0,$12 ; 0,24$ e $0,36 \%$ de $\mathrm{P}$ na dieta total, respectivamente. A suplementação com fósforo causou aumento linear na excreção de fósforo fecal com o aumento do fósforo consumido, fato reportado também por Scott et al. (1985) e Challa \& Braithwaite (1988).

O metabolismo mineral em ruminantes envolve grande complexidade com interação entre vários elementos macro e microminerais (Underwood, 2001). González (2000) afirmou que a ingestão excessiva de cálcio e fósforo pode diminuir a absorção intestinal de outros minerais, como magnésio, manganês e cobre.

Equações de regressão para estimativa do consumo de fósforo por meio da quantificação de fósforo excretado nas fezes têm sido determinadas em bovinos a pasto (Moir, 1960; Cohen, 1974; Holechek et al., 1985), no entanto, são raras as pesquisas realizadas especificamente com bubalinos. Esses autores obtiveram diferentes equações de regressão por várias causas, entre elas, raça, categoria animal e tipos de forrageiras ingeridas. Agarwala et al. (1971) estudaram as exigências de fósforo para bubalinos em mantença utilizando três fontes de fósforo (fosfato dihidrogênio de sódio, fosfato bicálcico e fósforo contido no farelo de trigo) e estimaram exigências diárias de 12 a $17 \mathrm{~g}$.

Com este estudo, objetivou-se estudar o balanço de macrominerais ( $\mathrm{P}, \mathrm{Ca}, \mathrm{Mg}, \mathrm{Na}, \mathrm{Ke}$ S) por meio de avaliações da ingestão e da concentração no rúmen e nas fezes em búfalos mantidos com dietas com diferentes níveis de fósforo (8, 12, 15 ou $18 \mathrm{~g} /$ animal.dia).

\section{Material e Métodos}

Quatro búfalos (Bubalus bubalis) da raça Mediterrâneo, machos não-castrados, com peso médio inicial de $635 \mathrm{~kg}$ e cânulas no rúmen foram mantidos em baias individuais contendo bebedouro automático no Setor Experimental Animal da Faculdade de Zootecnia e Engenharia de Alimentos, Campus da USP de Pirassununga. A colocação das cânulas de latex (10 cm diâmetro) no rúmen foi realizada por procedimento cirúrgico, seguido de adaptação e manutenção higiênica e sanitária. Antes do início do experimento, os animais receberam vermífugo e injeção intramuscular de complexo vitamínico A, D e E.

Os búfalos foram delineados em experimento quadrado latino $(4 \times 4)$ com subperíodos experimentais de 28 dias, compreendendo 14 dias de adaptação e 14 dias de coleta de dados. As dietas foram formuladas à base de cana-deaçúcar como volumoso e concentrado contendo fosfato bicálcico nos níveis de $0,1,3$ ou 5\% para proporcionar ingestão de fósforo de 8, 12, 15 e 18 g/dia (Tabelas 1 e 2). $O$ concentrado basal foi composto de ingredientes pobres em fósforo: levedura desidratada (20\%), polpa cítrica (37\%), gérmen de milho (30\%) e uréia (8\%). A cana-de-açúcar foi colhida a cada dois dias e picada mecanicamente no momento do fornecimento aos animais, duas vezes ao dia (às 8 e 16 h), na quantidade aproximada de 20 kg de matéria original por animal e $1 \mathrm{~kg}$ de concentrado, mantendo-se o controle diário das sobras. Análises bromatológicas para

Tabela 1 - Teores médios de matéria seca, proteína bruta, fibra em detergente neutro, energia bruta e de macrominerais das dietas

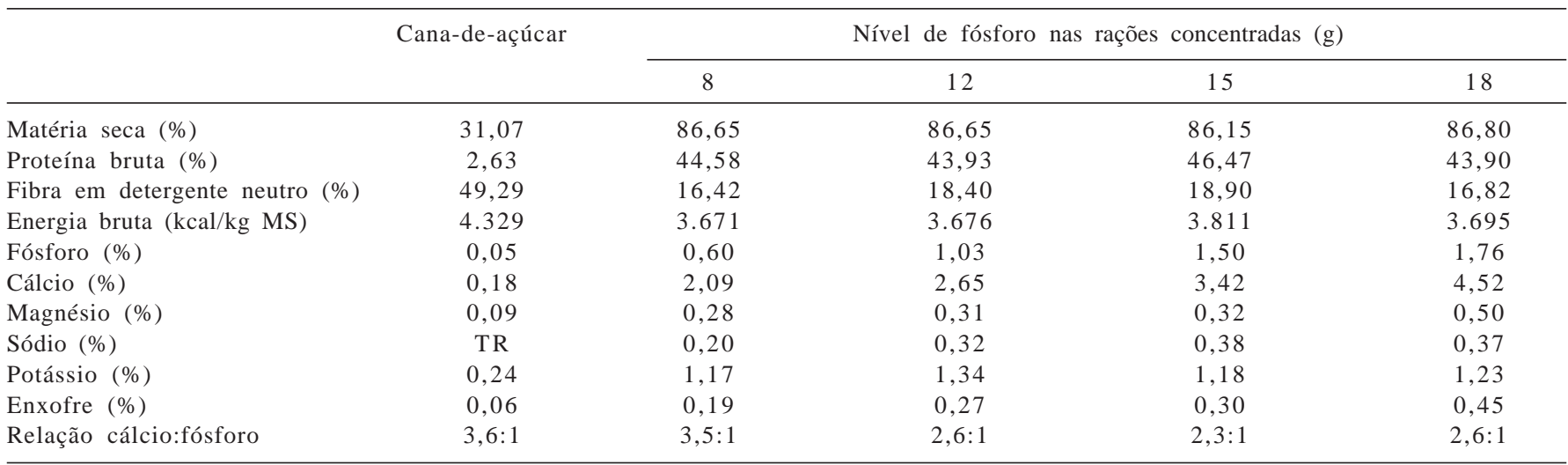


Tabela 2 - Ingestão de proteína bruta, fibra em detergente neutro e macrominerais ( $\mathrm{g} / \mathrm{kg}$ MS) em bubalinos alimentados com rações com níveis crescentes de fósforo

\begin{tabular}{lcccc}
\hline & \multicolumn{4}{c}{ Nível de fósforo na dieta (g) } \\
\cline { 2 - 5 } & 8 & 12 & 15 & 18 \\
\hline Proteína bruta (g/kg MS) & 78,7 & 92,4 & 93,6 & 96,2 \\
Fibra em detergente neutro (\%) & 437,5 & 446,1 & 457,5 & 445,1 \\
Fósforo (\%) & 1,2 & 2,1 & 2,9 & 3,4 \\
Cálcio (\%) & 4,2 & 5,8 & 7,1 & 9,2 \\
Magnésio (\%) & 1,1 & 1,3 & 1,3 & 1,6 \\
Sódio (\%) & 0,3 & 0,5 & 0,6 & 0,6 \\
Potássio (\%) & 3,6 & 4,2 & 3,9 & 4,1 \\
Enxofre (\%) & 0,7 & 0,9 & 0,9 & 1,2 \\
Relação cálcio:fósforo & 3,5 & 2,8 & 2,5 & 2,7 \\
\hline
\end{tabular}

utilização nos cálculos de ingestão de nutrientes e minerais foram realizadas de acordo com procedimentos descritos por Silva \& Queiroz (2002).

Amostras de conteúdo ruminal foram obtidas de três diferentes locais no rúmen, constituindo amostra única de cada animal coletada no 28o dia de cada subperíodo. Foram realizadas ainda medidas repetidas no tempo, correspondentes às coletas de conteúdo ruminal ao longo do dia, com amostragens nos tempos 0, 2, 8, e 10 h após a alimentação da manhã. As amostras de cada tempo foram colocadas em sacos plásticos, devidamente etiquetadas e congeladas a $-10^{\circ} \mathrm{C}$.

Amostragens de fezes foram feitas diariamente nos períodos da manhã ( 8 h) e da tarde (16 h) nos últimos seis dias de cada subperíodo experimental. As fezes foram retiradas diretamente do reto do animal e acondicionadas em um único saco plástico por período por animal, em porções de aproximadamente 150 g por coleta, e imediatamente armazenadas em congelador a $-10^{\circ} \mathrm{C}$.

No final de cada subperíodo, as amostras de conteúdo ruminal e de fezes foram descongeladas em temperatura ambiente e colocadas em bandejas de alumínio para présecagem em estufa de ventilação forçada a $65^{\circ} \mathrm{C}$ por 72 horas. Em seguida, foram trituradas em moinho tipo Willey com peneira de $1 \mathrm{~mm}$ e acondicionadas em saquinhos plásticos para posterior análise dos teores de $\mathrm{P}, \mathrm{Ca}, \mathrm{Mg}, \mathrm{Na}, \mathrm{K}$ e S. As determinações de minerais foram realizadas conforme técnicas descritas por Silva \& Queiroz (2002) em solução mineral preparada por digestão em via úmida. Após as devidas diluições, a concentração de fósforo foi determinada por colorimetria; a de enxofre pelo método turbidimetria do sulfato de bário; cálcio e magnésio em espectrofotometria de absorção atômica; e as concentrações de sódio e de potássio em espectrofotometria de chama.

A taxa de passagem do líquido ruminal foi estimada pela adição de 100 g de polietilenoglicol (PEG) como marcador no rúmen de cada animal e pela determinação da concentração do marcador 0, 1 e 24 horas depois, pelo método colorimétrico de turbidimetria (Hyden, 1955). Após as regressões e os cálculos para determinação da taxa de passagem de líquidos e do volume no rúmen, foram realizados cálculos para determinação da quantidade de conteúdo ruminal e estimativa da taxa de passagem de sólidos, de acordo com Alexander et al. (1969) e Waner \& Stacy (1968), utilizando-se as fórmulas: Total de água no rúmen $=V R / Y$, em que $V R=$ volume ruminal determinado pelo PEG e Y = porcentagem de água disponível, estimada pela equação de regressão desenvolvida para volumosos: $\mathrm{Y}=96,10-1,239 \mathrm{X}$, em que $\mathrm{X}=\%$ matéria seca. A quantidade total de conteúdo no rúmen foi estimada como o Total de conteúdo ruminal = total de água no rúmen/\% água; $\mathrm{e}$ a taxa de passagem de sólidos = kg MS ingerida por hora $/ \mathrm{kg}$ MS total no rúmen. O teor de matéria seca do conteúdo ruminal foi determinado pela secagem de amostras em estufa durante 24 horas a $100^{\circ} \mathrm{C}$.

Os dados obtidos foram analisados em programa estatísitico computacional (SAS, 1985) e as médias foram testadas pelo teste Tukey com nível de significância de $5 \%$. Os dados da concentração mineral no conteúdo ruminal foram adicionados do fator medidas repetidas no tempo, referentes aos momentos de colheita entre as refeições, e a análise foi realizada utilizando-se o comando "repeated”, gerado pelo procedimento do módulo GLM (PROC GLM do SAS). A separação por contrastes foi baseada na média de todos os tempos de amostragem.

\section{Resultados e Discussão}

Os animais apresentaram baixo consumo médio diário de matéria seca $\left(45,85 \mathrm{~g} / \mathrm{kg}^{0,75}\right)$, o que pode ser atribuído à elevada proporção de cana-de-açúcar, volumoso de baixa qualidade na dieta ( $85,5 \%$ da MS).

Durante o período experimental, verificou-se variação no consumo individual das dietas (Tabela 2), o que está relacionado à espécie animal, principalmente com animais mantidos em baias. Essa variação promoveu pequenas diferenças nas ingestões finais dos nutrientes, com exceção de fósforo e de cálcio. Apesar da fonte de fósforo utilizada (o fosfato bicálcico também é uma fonte de cálcio), a relação cálcio:fósforo manteve-se próxima entre os níveis de fósforo testados e variou de 2,5:1 a 3,5:1.

As concentrações dos macrominerais ( $\mathrm{P}, \mathrm{Ca}, \mathrm{Mg}, \mathrm{Na}, \mathrm{K}$ e S) não diferiram significativamente $(P>0,05)$ entre os níveis de fósforo (Tabela 3). A concentração média de fósforo no conteúdo ruminal foi de $0,98 \%$ da matéria seca, enquanto o teor de fósforo nas rações variou de 0,12 a 0,34\% e foi 8, 5, 3 e 3 vezes maior nos níveis de 8, 12, 15 e 
Tabela 3 - Concentrações de macrominerais no conteúdo ruminal, em quatro tempos de amostragem, em bubalinos alimentados com rações com níveis crescentes de fósforo

\begin{tabular}{|c|c|c|c|c|}
\hline \multirow{2}{*}{$\begin{array}{l}\text { Tempo após } \\
\text { alimentação (horas) }\end{array}$} & \multicolumn{4}{|c|}{ Nível de fósforo na dieta (g) } \\
\hline & 8 & 12 & 15 & 18 \\
\hline & \multicolumn{4}{|c|}{ Fósforo (\%) } \\
\hline 0 & 1,02 & 1,08 & 1,04 & 0,99 \\
\hline 2 & 0,95 & 0,93 & 0,95 & 0,99 \\
\hline 8 & 0,84 & 0,84 & 0,83 & 0,88 \\
\hline 10 & 1,06 & 1,12 & 1,07 & 1,13 \\
\hline \multirow[t]{2}{*}{ Média } & 0,97 & 0,99 & 0,97 & 1,00 \\
\hline & \multicolumn{4}{|c|}{ Cálcio (\%) } \\
\hline 0 & 0,34 & 0,40 & 0,42 & 0,35 \\
\hline 2 & 0,33 & 0,30 & 0,31 & 0,31 \\
\hline 8 & 0,24 & 0,25 & 0,27 & 0,31 \\
\hline 10 & 0,49 & 0,45 & 0,45 & 0,37 \\
\hline \multirow[t]{2}{*}{ Média } & 0,35 & 0,35 & 0,36 & 0,33 \\
\hline & \multicolumn{4}{|c|}{ Magnésio (\%) } \\
\hline 0 & 0,08 & 0,08 & 0,09 & 0,08 \\
\hline 2 & 0,08 & 0,08 & 0,08 & 0,08 \\
\hline 8 & 0,07 & 0,07 & 0,08 & 0,08 \\
\hline 10 & 0,10 & 0,09 & 0,09 & 0,10 \\
\hline \multirow[t]{2}{*}{ Média } & 0,08 & 0,08 & 0,09 & 0,08 \\
\hline & \multicolumn{4}{|c|}{ Sódio (\%) } \\
\hline 0 & 1,84 & 2,04 & 2,01 & 1,94 \\
\hline 2 & 1,35 & 1,43 & 1,37 & 1,41 \\
\hline 8 & 2,02 & 2,04 & 2,01 & 2,04 \\
\hline 10 & 0,95 & 0,95 & 0,98 & 1,05 \\
\hline \multirow[t]{2}{*}{ Média } & 1,54 & 1,61 & 1,59 & 1,61 \\
\hline & \multicolumn{4}{|c|}{ Potássio (\%) } \\
\hline 0 & 0,97 & 1,01 & 1,04 & 0,96 \\
\hline 2 & 1,81 & 1,81 & 1,90 & 1,85 \\
\hline 8 & 0,75 & 0,73 & 0,74 & 0,77 \\
\hline 10 & 2,25 & 2,23 & 2,19 & 2,28 \\
\hline \multirow[t]{2}{*}{ Média } & 1,45 & 1,45 & 1,47 & 1,46 \\
\hline & \multicolumn{4}{|c|}{ Enxofre (\%) } \\
\hline 0 & 0,09 & 0,10 & 0,09 & 0,09 \\
\hline 2 & 0,08 & 0,08 & 0,08 & 0,08 \\
\hline 8 & 0,09 & 0,09 & 0,09 & 0,10 \\
\hline 10 & 0,11 & 0,12 & 0,11 & 0,11 \\
\hline Média & 0,09 & 0,10 & 0,09 & 0,09 \\
\hline
\end{tabular}

Não houve diferenças significativas $(\mathrm{P}>0,05)$ nos valores médios entre os níveis de fósforo.

18 g, respectivamente. Essa diferença pode ser explicada principalmente pela reciclagem do fósforo pela saliva, comprovando que, em situações de baixa ingestão de fósforo ( $8 \mathrm{~g} / \mathrm{animal} / \mathrm{dia}$ ), há maior reciclagem de fósforo no organismo, elevando a concentração no rúmen, via saliva. Dessa forma, o hospedeiro estaria mobilizando fósforo armazenado no osso, uma vez que, segundo Underwood (1979), em ruminantes a mobilização de fósforo é mais importante que o fósforo dietético em determinadas condições de produção e saúde.
As concentrações de sódio e de potássio no conteúdo ruminal variaram inversamente nos tempos antes dos arraçoamentos e 2 horas após, respectivamente. Assim, em 8 horas após o arraçomento da manhã, a concentração média de sódio no conteúdo ruminal foi de $2,03 \%$ da MS e a de potássio $0,75 \%$ da MS e, em 2 horas após arraçoamento da tarde, os teores de sódio e potássio foram em média de 0,98\% e 2,24\% da MS, respectivamente. Essa variação provavelmente reflete no fluxo salivar no rúmen, uma vez que, segundo Bailey \& Balch (1961), o sódio e o potássio respondem inversamente à taxa de secreção salivar, ou seja, com o aumento da taxa de secreção salivar, a concentração de sódio se eleva e a de potássio diminui na saliva e vice-versa.

A dinâmica no rúmen comprovou rápida taxa média de passagem do líquido ruminal e lenta taxa de passagem de sólidos (Tabela 4), sem diferenças significativas ( $\mathrm{P}>0,05)$ entre os níveis de fósforo. Também não houve diferença significativa $(\mathrm{P}>0,05)$ no volume ruminal em litros $(\mathrm{L})$ e em $\mathrm{mL} / \mathrm{kg}^{0,75}$ entre os níveis de ingestão de fósforo pelos animais. Milton \& Ternouth (1985) também não verificaram diferença na taxa de passagem de líquido e no volume ruminal ao avaliarem o metabolismo de fósforo em ruminantes. A taxa de passagem média de sólidos no rúmen de $0,02 \mathrm{~h}^{-1}$ refletiu o nível alto de volumoso na dieta e a baixa ingestão de matéria seca pelos animais, corroborando informações citadas pelo ARC (1980) para ruminantes consumindo volumoso de baixa qualidade.

Mesmo com baixas ingestões de fósforo, a quantidade desse mineral no conteúdo ruminal foi muito alta, o que não ocorreu com os demais minerais (Tabela 5), o que comprova a grande importância da reciclagem do fósforo pela saliva para atender à demanda microbiana no rúmen por esse nutriente. No nível de ingestão diária de $8 \mathrm{~g}$ de fósforo, os valores de excreção de fósforo nas fezes foram maiores que os de fósforo ingerido, resultando na disponibilidade aparente negativa de $11,31 \%$, o que demonstra fluxo mais elevado de microrganismos do rúmen na presença de fósforo, bem como descamação e perda endógena de fósforo de células nas fezes. De fato, de acordo com Underwood (1999), as fezes são a principal rota de excreção de fósforo em animais ruminantes em pastejo ou alimentados com rações ricas em volumoso, enquanto a via urinária é de maior importância em monogástricos ou em ruminantes alimentados com rações ricas em concentrados ou com volumosos processados que diminuem a produção de saliva. Esse mecanismo poderia garantir a mantença do animal por um período de tempo em deficiência dietética de fósforo com o hospedeiro utilizando o fósforo de microrganismos do rúmen. De acordo com Witt \& Owens (1983), em animais jovens em crescimento, esse déficit pode ser mais grave. 
Tabela 4 - Taxas de passagem de líquido e de sólidos no rúmen e volume ruminal em bubalinos alimentados com rações com níveis crescentes de fósforo

\begin{tabular}{|c|c|c|c|c|}
\hline & \multicolumn{4}{|c|}{ Nível de fósforo da dieta (g/dia) } \\
\hline Taxa de passagem de líquido (L/hora) & 0,096 & 0,090 & 0,092 & 0,086 \\
\hline Taxa de passagem de sólidos (g/hora) $)^{1}$ & 0,022 & 0,020 & 0,020 & 0,018 \\
\hline Taxa de passagem de sólidos (g/dia) ${ }^{1}$ & 0,53 & 0,47 & 0,49 & 0,42 \\
\hline Volume ruminal (L) & 59,14 & 70,10 & 61,31 & 74,99 \\
\hline
\end{tabular}

Não houve diferenças significativas ( $\mathrm{P}>0,05)$ nos valores médios entre os níveis de fósforo em qualquer parâmetro (mesma linha).

${ }^{1}$ Estimativas calculadas a partir da taxa de passagem de líquido ruminal e do teor de matéria seca no rúmen.

Tabela 5 - Ingestão, quantidade no rúmen, excreção nas fezes e disponibilidade aparente média de fósforo, cálcio e magnésio em bubalinos alimentados com rações com níveis crescentes de fósforo

\begin{tabular}{|c|c|c|c|c|}
\hline & \multicolumn{4}{|c|}{ Nível de fósforo da dieta (g/dia) } \\
\hline & \multicolumn{4}{|c|}{ Ingestão diária } \\
\hline Fósforo (g) & 7,78 & 11,66 & 15,26 & 17,99 \\
\hline Cálcio (g) & 26,55 & 31,69 & 37,32 & 48,13 \\
\hline \multirow[t]{2}{*}{ Magnésio (g) } & 6,65 & 7,05 & 6,68 & 8,79 \\
\hline & \multicolumn{4}{|c|}{ Quantidade total no conteúdo ruminal } \\
\hline Fósforo (g) & 102,52 & 124,74 & 112,48 & 135,98 \\
\hline Cálcio (g) & 37,81 & 45,53 & 40,66 & 45,55 \\
\hline \multirow[t]{2}{*}{ Magnésio (g) } & 8,76 & 10,02 & 9,73 & 11,66 \\
\hline & \multicolumn{4}{|c|}{ Quantidade diária excretada nas fezes } \\
\hline Fósforo（g) & $8,66 b$ & $11,59 a b$ & $13,99 a$ & $14,59 \mathrm{a}$ \\
\hline Fósforo (\%) & $-11,16 b$ & $0,63 b$ & $8,34 a b$ & $19,01 \mathrm{a}$ \\
\hline Cálcio (\%) & 44,48 & 38,47 & 44,94 & 38,83 \\
\hline Magnésio (\%) & 85,41 & 77,59 & 75,30 & 65,42 \\
\hline
\end{tabular}

Valores seguidos de letras diferentes na mesma linha diferem entre si $(\mathrm{P}<0,05)$ pelo teste Tukey.

Não houve diferença significativa $(\mathrm{P}>0,05)$ na disponibilidade aparente de cálcio e magnésio. Os valores de disponibilidade de magnésio foram maiores que os de cálcio e, embora não tenha diferido significativamente entre os níveis de fósforo estudados ( $\mathrm{P}>0,05)$, em valores absolutos, os valores de disponibilidade decresceram com o aumento da ingestão desse mineral.

A ingestão de níveis crescentes de fósforo $\left(\mathrm{g} / \mathrm{kg}^{0,75}\right)$ promoveu aumento linear $(\mathrm{P}<0,01)$ na excreção fecal desse mineral (g/kg0,75) (Figura 1), resultado semelhante aos obtidos por Holechek et al. (1985) em bovinos da raça Angus. Cohen (1974) observou regressão linear com coeficiente angular positivo de excreção de fósforo em bovinos da raça Hereford, enquanto neste estudo com búfalos, a quantidade de fósforo excretada pelos animais aumentou proporcionalmente à ingestão de fósforo (Tabela 6), corroborando os achados de Cohen (1974).

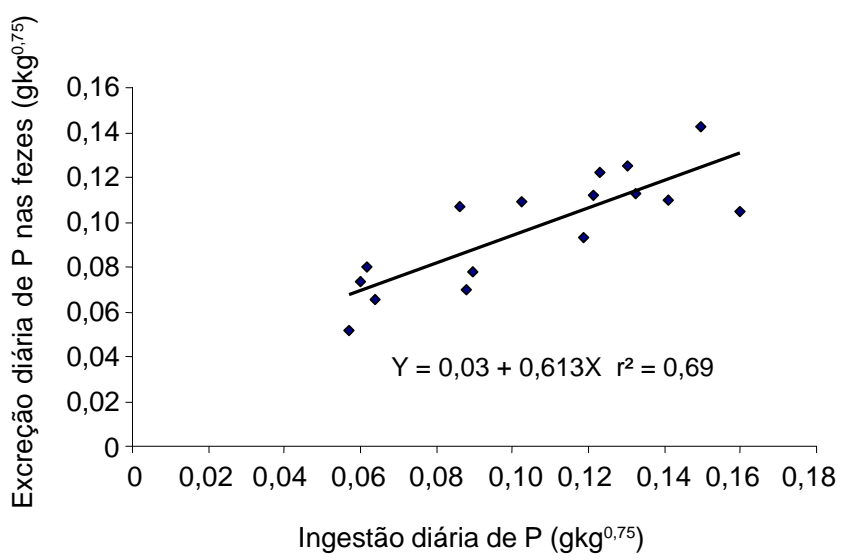

Figura 1 - Regressão linear da ingestão diária de fósforo (g/ $\mathrm{kg}^{0,75}$ ) como variável independente $(\mathrm{X})$ e excreção diária de fósforo nas fezes $\left(\mathrm{g} / \mathrm{kg}^{0,75}\right)$ como a variável dependente (Y) em bubalinos. 
Tabela 6 - Regressões lineares obtidas por diferentes autores usando fósforo ingerido $\left(\mathrm{g} / \mathrm{kg}^{0,75}\right)$ como variável independente $(\mathrm{X})$ e fósforo excretado nas fezes $\left(\mathrm{g} / \mathrm{kg}^{0,75}\right)$ como variável dependente (Y)

\begin{tabular}{lccc}
\hline Equação de regressão & Animal & ${ }^{*} \mathrm{n}$ & ${ }^{* *} \mathrm{r}^{2}$ \\
\hline $\begin{array}{l}\text { Presente trabalho } \\
\mathrm{Y}=0,03+0,613 \mathrm{X}\end{array}$ & Búfalos Mediterrâneo & 16 & 0,69 \\
$\begin{array}{l}\text { Holechek et al. (1985) } \\
\mathrm{Y}=0,20+0,69 \mathrm{X}\end{array}$ & Bovinos Angus & 33 & 0,71 \\
$\begin{array}{l}\mathrm{C} \text { ohen }(1974) \\
\mathrm{Y}=0,051+0,294 \mathrm{X}\end{array}$ & Bovinos Hereford & 11 & 0,96 \\
\hline${ }^{*} \mathrm{n}=$ número de observações; ${ }^{* *}{ }^{2}{ }^{2}=$ coeficiente de determinação. &
\end{tabular}

\section{Conclusões}

Baixo nível de fósforo na dieta de búfalos adultos promove alta reciclagem de fósforo no rúmen e disponibilidade negativa de fósforo. Níveis crescentes de fósforo na dieta na faixa de 8 a $18 \mathrm{~g} / \mathrm{animal} /$ dia não influenciam as disponibilidades de cálcio e magnésio. Ingestões de níveis crescentes de fósforo na dieta de 8 a $18 \mathrm{~g} / \mathrm{animal} / \mathrm{dia}$ promovem aumento linear na excreção fecal de fósforo e baixos valores de disponibilidade do fósforo, o que indica dietas deficientes desse elemento mineral para o metabolismo animal.

\section{Agradecimentos}

À empresa Premix - Técnica em Suplementação Animal, em Patrocínio Paulista-SP, pelo fornecimento das rações concentradas contendo diferentes níveis de fósforo.

\section{Literatura Citada}

AGARWALA, O.N.; NATH, K.; MAHADEVAN, V. Phosphorus requirement of buffalo. Journal of Agricultural Science, v.76, p.83, 1971.

AGRICULTURAL RESEARCH COUNCIL - ARC. The nutrient requirements of ruminant livestock. Slough: Commonwealth Agricultural Bureau, 1980. 351p.

ALEXANDER, C.L.; MEYER, R.M.; BARTLEY, E.E. Effect of quantity of rumen dry matter and other factors on determinations of rumen fluid volume with polyethylene glycol. Journal of Animal Science, v.29, p.69-74, 1969.
BAILEY, C.B.; BALCH, C.C. Saliva secretion and its relation to feeding in cattle. 1 . The composition and rate of secretion of parotid saliva in a small steer. British Journal of Nutrition, v.15, n.3, p.371-383, 1961.

BARCELLOS, J.O.J. O papel do fósforo na nutrição de bovinos de corte. In: DIAZ GONZALEZ, F.H.; OSPINA, H.; BARCELLOS, J.O.J. (Eds.). Nutrição mineral em ruminantes. 2.ed. Porto Alegre: Editora da UFRGS, 1998. p.23-72.

CHALLA, J.; BRAITHWAITE, G.D. Phosphorus and calcium metabolism in growing calves with special emphasis on homeostasis. 1. Studies of the effects of changes in the dietary phosphorus intake and calcium metabolism. Journal of Agricultural Science, v.110, n.3, p.573-581, 1988.

COHEN, R.D.H. Phosphorus nutrition of beef cattle. 4. The use of fecal and blood phosphorus for the estimation of phosphorus intake. Australian Journal Experimental Agricultural Animal Husbandry, v.14, p.709-715, 1974.

GONZÁLEZ, F.H. Indicadores sanguíneos do metabolismo mineral em ruminantes. In: DIAZ GONZÁLEZ, F.H.; BARCELLOS, J.O.; RIBEIRO, L.A.O. (Eds.) Perfil metabólico em ruminantes: seu uso em nutrição e doenças nutricionais. Porto Alegre: UFRGS, 2000. p.31-51.

HOLECHEK, J.L.; GALYEAN, M.L.; WALLACE, J.D. et al. Evaluation of fecal indices for predicting phosphorus status of cattle. Grass and Forage Science, v.40, p.489-492, 1985.

HYDEN, S. A tubidimetric method for determination of higher poliethilene glycol in biological materials. Kungl Lanthbrukshogskolans Annaler, v.22, p.139-145, 1955.

MILTON, J.T.B.; TERNOUTH, J.H. Phosphorus metabolism in ruminants. II. Effects of inorganic phosphorus concentration upon food intake and digestibility. Australian Journal of Agricultural Research, v.36, p.647-654, 1985.

MOIR, K.B. Nutrition of grazing cattle. 2. Estimation of phosphorus and calcium in pasture selected by grazing cattle. Queensland Journal Agricultural Science, v.17, p.373-383, 1960.

STATISTICAL ANALYSIS SYSTEM - SAS. SAS user's guide: statistics. Cary: SAS Institute, 1985. v.5.

SCOTT, D.; WHITELAW, F.G.; BUCHAN, W. et al. The effect of variation in phosphorus intake on salivary phosphorus secretion, net intestinal phosphorus absorption and faecal endogenous phosphorus excretion in sheep. Journal of Agricultural Science, v.105, p.271-277, 1985.

SILVA FILHO, J.C.; VITTI, D.M.S.S.; CAMPOS NETO, O. et al. Exigência mínima de fósforo em novilhos da raça Nelore. Pesquisa Agropecuária Brasileira, v.35, n.9, p.1861-1865, 2000.

SILVA, D.J.; QUEIROZ, A.C. Análise de alimentos: métodos químicos e biológicos. 3.ed. Viçosa, MG: Universidade Federal de Viçosa, 2002. 235p.

TORKANIA, C.H.; DOBEREINER, J.; PEIXOTO, P.V. Deficiências minerais em animais de fazenda, principalmente bovinos em regime de campo. Pesquisa Veterinária Brasileira, v.20, n.3, p.127-138, 2000.

UNDERWOOD, E.J.; SUTTLE, N.F. The mineral nutrition of livestock. 3.ed. Wallingford: CABI Publishing, 2001. 614p.

WANER, A.C.I.; STACY, B.C. The fate of water in the rumen. British Journal of Nutrition, v.22, p.369-387, 1968.

WITT, K.E.; OWENS, F.N. Phosphorus: ruminal availability and effects on digestion. Journal of Animal Science, v.56, n.4, p.930-937, 1983. 